REVIEW

\title{
From hemp seed and porcupine quill to HRCT: advances in the diagnosis and epidemiology of bronchiectasis
}

\section{A Spencer}

Arch Dis Child 2005;90:712-714. doi: 10.1136/adc.2004.054031

Over the last decade there has been a significant improvement in our ability to recognise non-cystic fibrosis (CF) bronchiectasis in children. The precise incidence is uncertain, and it varies greatly depending on the populations studied and the methods used to make the diagnosis. It is unlikely that many of the underlying causes of non-CF bronchiectasis will be eradicated in the near future, and so it must be expected that with ever improving technology this diagnosis will be made with increasing frequency. This emphasises the need to improve our understanding of the aetiology, pathophysiology, epidemiology, and management options for children with this group of conditions.

Correspondence to: Dr D A Spencer, Regional Cardiothoracic Centre, Freeman Hospital Newcastle upon Tyne NE7 7DN, UK; david.spencer@ nuth.northy.nhs.uk

Accepted 13 March 2005
$\mathrm{T}$ he first description of bronchiectasis was famously made by Laënnec, who in 1819 used various objects including seeds and quills to define the calibre of abnormally dilated bronchi. ${ }^{1}$ The declining incidence of traditional causes of non-cystic fibrosis (CF) bronchiectasis such as tuberculosis and pertussis has led to repeated suggestions that this problem is now so uncommon that it should be considered as an "orphan" disease. ${ }^{2}$ However, with increased use of high resolution computed tomography (HRCT) over the last decade many more children are now being diagnosed with this problem. ${ }^{3}$ Given these uncertainties it is appropriate to reconsider the significance of bronchial dilatation in children with suppurative lung disease.

Estimates of the incidence of this entity in Western type populations are increasing progressively as ever more sensitive methods are introduced into routine use, although many of these "new" cases will tend to be found at the milder end of the disease spectrum. In this edition of Archives (see page 737) Twiss et al describe the results of a national survey of paediatricians from New Zealand which suggests that the incidence of non-CF bronchiectasis in this diverse population is in the region of 3.7 per 100000 with a prevalence of 1 in $3000 .{ }^{4}$ The authors suggest that overall prevalence rates for non-CF bronchiectasis are higher than for $\mathrm{CF}$, even though a significant proportion of the population clearly derives from European stock in whom CF has until now been regarded as the dominant cause of bronchiectasis in children. They report a much higher incidence of bronchiectasis in at-risk groups such as Maoris and Pacific Island children resident in New Zealand in whom a rate of up to 19.4 per 100000 has been estimated. This increased risk of disease seen in certain ethnic groups is comparable to that seen in other indigenous populations such as Aborigines and Inuits. ${ }^{5}$ Our data from the North East of England demonstrate a comparable overall prevalence of 1 in 5800 in children, ${ }^{3}$ although this figure may be slightly higher than found in other developed populations because of the inclusion of at risk groups such as transplant recipients and patients with complex immunological problems. It is most likely that all of these quoted figures still represent a significant under diagnosis of the problem, and further prospective epidemiological studies will be required to gain an accurate insight in to the true incidence and prevalence of this entity in different populations. It is also of concern that in our experience this condition is currently associated with significant diagnostic delay, with the median interval between onset of respiratory symptoms and diagnosis being three years. ${ }^{3}$

The dominant symptom is almost invariably chronic cough, and many patients are referred with so called "cough variant asthma" which has not responded to anti-asthma medications. It is now appreciated that the great majority of true asthmatics will wheeze, and that cough in the absence of wheeze is unlikely to be due to asthma. There is now considerable doubt as to whether cough variant asthma even exists in children. ${ }^{6}$ This increasing reluctance to label the child whose only respiratory symptom is cough as having asthma has led to investigations being performed much more frequently in this group of patients, and has in turn revealed that previously unrecognised chronic bacterial endobronchial infection is relatively common.

The basic diagnostic algorithm includes obtaining specimens of airway secretions for bacterial culture, radiological investigations, sweat test, and immune function tests. Other investigations may clearly be required according to the clinical picture. Most young children will not expectorate sputum and so cough swab or nasopharyngeal aspirate are frequently required. Interpretation of the significance of these findings can sometimes be difficult as the most common pathogens isolated are $H$ influenzae and $S$ pneumococcus which may also be upper airway commensals. Bronchoscopy is a useful tool which may reveal evidence of previously unrecognised endobronchial infection and also helps

Abbreviations: CAP, community acquired pneumonia; $C F$, cystic fibrosis; $H R C T$, high resolution computed tomography; PCD, primary ciliary dyskinesia. 
to differentiate between upper airway colonisation and true endobronchial infection.

Radiological changes have long been reported in bronchiectasis, initially using plain chest radiography and bronchography, followed by plain computed tomography (CT) and more recently high resolution CT (HRCT). ${ }^{78}$ The plain chest radiograph is of very limited use, as it lacks sensitivity and may be totally normal even in children with significant bronchiectatic changes on HRCT. ${ }^{9}$ HRCT is now accepted as the "gold standard" for making the diagnosis, with changes demonstrated correlating reasonably well with histological changes found after lobectomy in adults. ${ }^{10}$ Our knowledge of the relations between pathological processes, histological changes, and radiological abnormalities in children is necessarily incomplete as it is now uncommon to obtain biopsy material from children with suppurative lung disease, whereas HRCT technology continues to advance and detect increasingly subtle abnormalities.

\section{PATHOPHYSIOLOGY}

The microscopic changes of bronchiectasis were first documented in the 1940s and 50s. ${ }^{11}{ }^{12}$ Bronchial dilatation is associated with loss of cilia, cubiodal and squamous metaplasia, hypertrophy of bronchial glands and lymphoid hyperplasia, along with marked vascular changes. It has been recognised for many years that these changes are intimately related to chronic bacterial infection, and several theories have been advanced to explain this relation. The concept of the "vicious cycle"13 proposed by Peter Cole and colleagues from the Brompton Hospital has been largely accepted over the last two decades. According to this theory, chronic bacterial endobronchial infection results in inflammation and airway damage causing bronchial dilatation. It is proposed that airway dilatation results in mucociliary statis, which in turn promotes further bacterial infection, increased airway inflammation, and more bronchial dilatation. Intrinsic to this theory is the concept that bronchial dilatation is both permanent and progressive without treatment. Although this concept has many attractions and appears to hold good for most patients with established suppurative lung disease, recent advances suggest that revision is now required. This is particularly important when considering the relations between infection and repair in the developing lung, and that HRCT changes may be subtle.

It has been recognised for many years that bronchial dilation seen during an acute pneumonic illness may resolve. Similar changes seen in the context of an inhaled foreign body may also regress after removal of the offending object. ${ }^{14}{ }^{15}$ However, until recently it has been thought that bronchial dilation seen in the context of chronic suppurative lung disease was permanent. It is only with the increased use of repeated HRCT scans performed over prolonged periods of time that it has become apparent that HRCT changes may not always progress as would be predicted from the vicious cycle theory. ${ }^{16}$ In many patients radiological changes remain static over several years and in some there may be improvement or even complete resolution. ${ }^{3}{ }^{16}$ It is not known if this is due to the beneficial effects of early aggressive treatment with antibiotics and physiotherapy, or whether some patients with milder disease may sometimes have a self limiting problem even without treatment. Randomised controlled trials would clearly be required to answer this question with certainty, but ethical considerations suggest that it would not now be possible to perform such studies. These observations imply that the term bronchiectasis, which is associated with both permanence and progression, is no longer appropriate for all of our patients. We have suggested that the term should be broadened in to two categories: "HRCT bronchiectasis" and "established bronchiectasis". ${ }^{3}$ HRCT bronchiectasis might be used to describe changes seen on a single scan which may resolve, remain static, or progress. The label of established bronchiectasis would refer to changes that persist or progress on repeat scans performed over a significant period of timewe have suggested two years. Many children with chronic endobronchial infection have non-specific changes such as bronchial wall thickening on HRCT. Although little is known of the natural history of these changes, they certainly proceed to bronchiectasis in some children, and we have suggested that this entity might be referred to as "prebronchiectasis". Although these children certainly have a form of chronic bronchitis, it is probably best to avoid this actual term as it is more closely associated with chronic obstructive pulmonary disease seem in adult smokers.

\section{AETIOLOGY}

Previous studies have reported that an underlying cause for bronchiectasis can be determined in about $70 \%$ of patients, with the remainder being labelled as idiopathic. ${ }^{17}$ With improving diagnostic techniques the proportion of idiopathic patients may change, especially with recognition of more subtle immunological abnormalities such as functional antibody deficiency ${ }^{18}$ and improving facilities for the assessment of primary ciliary dyskinesia (PCD).

PCD is a rare but important cause of bronchiectasis because early diagnosis and treatment appears to improve the prognosis. ${ }^{19}$ It is also likely that a genetic diagnosis may soon be feasible in at least a proportion of affected families. ${ }^{20}$ It needs to be appreciated though that this group of conditions is very uncommon, and that this diagnosis was only confirmed in $20 \%$ of children with established idiopathic suppurative lung disease referred to a supraregional ciliary dyskinesia service for assessment ( $\mathrm{O}^{\prime}$ Callaghan $\mathrm{C}$, personal communication). This indicates that we need to continue searching vigorously for further diagnoses in order to reduce the proportion of idiopathic cases. ${ }^{3}{ }^{17}$

A significant proportion of the idiopathic group may be patients who have suffered damage as a consequence of community acquired pneumonia (CAP). Studies on the effect of pneumonic illness on the development of bronchiectasis are complicated by the fact that there is often a considerable delay between the acute illness and the recognition of chronic suppurative lung disease. It can also be difficult to determine with certainty whether the child was completely well before the acute illness or whether there may have been a preexisting problem responsible for the development of the pneumonia. It is well recognised that children in at risk groups such as American Indians and native Alaskan children $^{5}$ are prone to develop bronchiectasis in association with pneumonic illness, but whether this is because of the consequences of repeated infections, genetic predisposition, nutritional, or socioeconomic factors is not always clear. The relevance, if any, of these findings to the development of bronchiectasis in children living in more "developed" societies is also uncertain. However, it has recently been shown in a large case control series that children hospitalised for CAP in the UK do develop more bronchiectasis than controls, and that this problem is frequently associated with obliterative bronchiolitis. ${ }^{21}$ As most post-infectious obliterative bronchiolitis occurs after adenoviral infection, this suggests that viral studies should be included as a routine component of the investigation of children hospitalised for CAP. Such a policy may reduce the diagnostic delay frequently seen in these patients.

\section{TREATMENT}

The lack of clinical trial data in this area renders it difficult to produce rigid evidence based management guidelines. The treatment of any underlying medical problems clearly needs 
to be optimised, and in particular the management of any possible immune deficiency requires specialist input from a paediatric immunologist. All children should receive the influenza vaccine annually. Use of polysaccharide and conjugate pneumococcal vaccines should ideally be dictated by the results of pneumococcal antibody titres and the response to these vaccines monitored as part of the immunological assessment. ${ }^{18}$

Surgical options are limited because many patients have generalised disease. Lobectomy may be curative for a small number of patients in whom disease is limited to a single lobe and difficult to control by medical means alone.

Antibiotics are a central component of treatment. Choice of antibiotic is usually relatively straightforward, as by far the most common organism isolated in children is $H$ influenzae, although this will usually be of a non-capsulated form that cannot be prevented by vaccination. Given the serious nature of the condition and the potential for progression there is logic in using high doses and prolonged courses of treatment, but given the lack of trial data such decisions must be determined by the clinical condition and response to therapy. Fortunately, persistent infection with difficult organisms such as $P$ aeruginosa is uncommon in paediatric practice. ${ }^{3}$ There are no available data on the use of nebulised antibiotics in this patient group.

The use of other therapies such as oral and inhaled corticosteroids, Rh DNase, and nebulised hypertonic saline remains speculative, and the best evidence available is based around case reports.

The role of physiotherapy in the management of children with non-CF bronchiectasis is still unclear. Although physiotherapeutic techniques have been demonstrated to be of benefit in improving sputum production and radiaerosol clearance in adults with non-CF bronchiectasis, ${ }^{22}$ the results of such studies cannot readily be extrapolated to children. ${ }^{23}$ Uncertainty relates both to the role of different therapeutic techniques as well as to the frequency with which therapy should be administered. In particular, there is a need to determine whether therapies need to be administered on a regular basis, or whether it is reasonable for treatment to be limited to periods of respiratory exacerbations. There are very reasonable concerns that non-administration of physiotherapy may be detrimental and therefore potentially unethical, but it must also be accepted that the some regimes have the potential to be not only ineffective but also positively harmful. ${ }^{23}$ It is also important to consider the detrimental social and emotional consequences of recommending time consuming and onerous treatments on the wellbeing of the child and family.

Treatment decisions are rendered more difficult because of the clinical diversity of non-CF bronchiectasis, but with the recognition of increasing numbers of patients and a greater willingness for collaboration between centres to perform randomised controlled trials, there is now a reasonable prospect that we can move towards beginning to answer some of these important questions. The advent of the British Paediatric Orphan Lung Disease project (see http:// www.bpold.co.uk) may be crucial in allowing sufficient cases to be identified nationally to allow such studies to be performed.

\section{CONCLUSIONS}

Over the last decade there has been a significant improvement in our ability to recognise non-CF bronchiectasis in children. It is unlikely that many of the underlying causes of this problem will be eradicated in the near future, and so it must be expected that with increasing awareness and ever improving technology this diagnosis will be made with increasing frequency. This emphasises the need to improve our understanding of the aetiology, pathophysiology, epidemiology, and management options for children with this group of conditions.

Competing interests: none declared

\section{REFERENCES}

1 Laënnec RTH. A treatise in the diseases of the chest and on mediate auscultation, 4th ed. London: Longman, 1819.

2 Callahan CW, Redding GJ. Bronchiectasis in children: orphan disease or persistent problem? Pediatr Pulmonol 2002;33:492-6.

3 Eastham KM, Fall AJ, Mitchell L, et al. The need to redefine non-cystic fibrosis bronchiectasis in childhood. Thorax 2004;59:324-7.

4 Twiss J, Metcalfe R, Byrnes CA. New Zealand national incidence of bronchiectasis "too high" for a developed country. Arch Dis Child 2005;90:736-40

5 Singleton R, Morris A, Redding G, et al. Bronchiectasis in Alaska Native children: causes and clinical courses. Pediatr Pulmonol 2000;29: 182-7.

6 McKenzie S. Cough-but is it asthma? Arch Dis Child 1994;70:1-2.

7 Swensen SJ, Aughenbaugh GL, Brown LR. High-resolution computed tomography of the lung. Mayo Clin Proc 1989;64:1284-94.

8 Kuhn JP, Brody AS. High-resolution CT of pediatric lung disease. Radiol Clin North Am 2002;40:89-110.

9 Fall AJ, Spratt J, Mitchell L, et al. Plain chest X-Ray vs high resolution CT (HRCT) in non-CF bronchiectasis (NCFB) in children. Thorax 2001;56/suppl 111):P26.

10 Kang EY, Miller RR, Muller NL. Bronchiectasis: comparison of preoperative thin-section CT and pathologic findings in resected specimens. Radiology 1995; 195:649-54.

11 Ogilvie AG. The natural history of bronchiectasis. Arch Intern Med $1941 ; 68: 68$

12 Whitwell $\mathbf{F}$. A study of the pathology and pathogenesis of bronchiectasis. Thorax 1952;7:213

13 Cole P. The damaging role of bacteria in chronic lung infection. J Antimicrob Chemother 1997;40(suppl A):5-10.

14 Mansour Y, Beck R, Danino J, et al. Resolution of severe bronchiectasis after removal of long-standing retained foreign body. Pediatr Pulmonol 1998;25:130-2.

15 Karakoç F, Karadag B, Akbenlioglu C, et al. Foreign body aspiration: what is the outcome? Pediatr Pulmonol 2002;25:130-2.

16 Gaillard EA, Carty H, Heaf D, et al. Reversible bronchial dilatation in children: comparison of serial high-resolution computer tomography scans of the lungs. Eur J Radiol 2003;47:215-20.

17 Nikolaizik WH, Warner JO. Aetiology of chronic suppurative lung disease [see comment]. Arch Dis Child 1994;70:141-2.

18 Wasserman RL, Sorensen RU. Evaluating children with respiratory tract infections:the role of immunization with bacterial polysaccharide vaccine. Pediatr Infect Dis J 1999; 18:157-63.

19 Ellerman A, Bisgaard $\mathrm{H}$. Longitudinal study of lung function in a cohort of primary ciliary dyskinesia. Eur Respir J 1997;10:2376-9.

20 Geremek M, Witt M. Primary ciliary dyskinesia: genes, candidate genes and chromosomal regions. J Appl Genet 2004;45:347-61.

21 Eastham KM, Parker L, Hammal D, et al. Community acquired pneumonia requiring admission to hospital is associated with chronic respiratory morbidity in childhood. Thorax 2004;59(suppl 11):S67

22 Jones $\mathrm{AP}$, Rowe $\mathrm{BH}$. Bronchopulmonary hygiene physical therapy in chronic obstructive pulmonary disease and bronchiectasis (Cochrane review). The Cochrane Library 1988:3.

23 Wallis C, Prasad A. Who needs chest physiotherapy? Moving from anecdote to evidence. Arch Dis Child 1999;80:393-7.

24 Button BM, Heine RG, Catto-Smith AG, et al. Chest physiotherapy, gastrooesophageal reflux, and arousal in infants with cystic fibrosis. Arch Dis Child 2004;89:435-9. 